\title{
Light amplification and localization in randomly layered media with gain
}

\author{
Zhao-Qing Zhang* \\ Department of Physics, Hong Kong University of Science and Technology, Clear Water Bay, Kowloon, Hong Kong
}

(Received 20 March 1995)

\begin{abstract}
Light amplification and localization behaviors in randomly layered media with gain are studied using the transfer-matrix method. It is found that localization is enhanced due to the amplification of coherent backscattering effect. It is also found that $\xi_{0}$ and $l_{g}$ are the only two relevant parameters in such systems, where $\xi_{0}$ is the localization length in the absence of gain and $l_{g}$ is the gain length. The interplay of $\xi_{0}$ and $l_{g}$ gives rise to two new lengths $L_{\mathrm{sr}}$ and $\xi$. $L_{\mathrm{sr}}$, which behaves like $\left(l_{g} \xi_{0}\right)^{0.5}$, is a manifestation of the threshold length for the super radiation, which is strongly suppressed in one dimension. $\xi$ is the localization length in the presence of gain, which can be described by $1 / \xi=1 / \xi_{0}+1 / l_{g}$. Results for dissipative media are also presented.
\end{abstract}

\section{INTRODUCTION}

Wave propagation in random media has been the focus of considerable study in the past decade. ${ }^{1}$ Recently, it has been reported that lasing is observed in strongly scattering media containing laser-active molecules. ${ }^{2}$ The cause of laser action in such nonresonant feedback media is believed to be related to the increase of photon path length due to multiple scattering. ${ }^{3}$ The theoretical consideration of laser action in random media actually appeared more than 25 years ago by Letokhov. ${ }^{4} \mathrm{He}$ considered the following diffusion equation with a gain term:

$$
\frac{\partial \phi(\mathbf{r}, t)}{\partial t}=D \nabla^{2} \phi(\mathbf{r}, t)+\frac{2 c}{l_{g}} \phi(\mathbf{r}, t),
$$

where $\phi$ is the photon density, $D$ is the diffusion constant, $c$ is the light speed, and $l_{g}$ is the gain length of the medium, which is twice the inverse of the gain coefficient. Using the relation between $D$ and the transport mean free path $l$, i.e., $D=c l / 3$, it is easy to see that the threshold value of $l_{g}$ for a nontrivial steady-state solution of Eq. (1) in a sample of size $L$ is on the order of $L^{2} / l$. In other words, in a medium with given values of $l_{g}$ and $l$ the critical size for a nontrivial solution of Eq. (1) is on the order of $\left(l l_{g}\right)^{0.5}$. Here, we denote this critical size by $L_{\mathrm{sr}}$ as the condition for a super radiation; i.e., $L_{\mathrm{sr}} \approx a_{d}\left(l l_{g}\right)^{0.5}$, where $a_{d}$ is a geometrical factor on the order of 1. Physically, this condition requires that the path length of a photon traveling across a sample of size $L$ be greater than the gain length $l_{g}$ in order to have a super-radiant emission. Here we use the term "super radiation" to denote the occurrence of lasing in random media. Thus, in the case of nonresonant feedback, the entire spectrum of modes is eligible for super radiation. However, the localization effect has been ignored in the above discussions. In systems where the coherent backscattering is important, the presence of gain in the media may also enhance the coherent backscattering effect, which in turn could suppress the super radiation and make a localized state more localized. In the case of weak localization, recent studies have shown that the amplification of the back- scattered light could drastically narrow the enhanced backscattering peak. ${ }^{5}$

The effects due to localization are best understood in one dimension. It is well known that in one dimension all states are localized. The localization length can be roughly estimated in the following way. Due to the localization effect, the maximally crossed diagrams give negative contributions $\delta D$ to the diffusion constant. ${ }^{6}$ In one dimension, $\delta D$ has the form $-(c / 2 \pi) \int_{q_{1}}^{q_{u} d q} / q^{2}$, where the upper cutoff $q_{u}$ is inversely proportional to the mean free path $l$, or $q_{u} \approx 1 / l$. While the lower cutoff $q_{1}$ is inversely proportional to the sample size $L$. If we define the localization length $\xi_{0}$ as the size where $\delta D \approx-D$, from $q_{1} \approx 1 / \xi_{0}$, we find $\xi_{0} \approx 3.1 l$. In the case of electronic systems, it has also been found that $\xi_{0} \approx(2-4) l$ in one dimension depending on various definitions of $l .{ }^{7}$ Since the localization length $\xi_{0}$ is only a few mean free paths, there does not actually exist a region of diffusion in one dimension and the super-radiant emission is largely suppressed. Instead of continuous modes, only a finite number of discrete modes are eligible for super radiation. Some interesting questions are the following. Although being suppressed, can the super-radiation threshold length $L_{\mathrm{sr}}$ manifest itself in other forms? If yes, will such a length $L_{\text {sr }}$ exist in the short-wavelength limit, where the mean free path $l$ is ill defined? Finally, how does the existence of the gain length $l_{g}$ alter the localization behavior in a quantitative way? To answer these questions, we have studied the light amplification and localization behaviors in one-dimensional (1D) randomly layered media by using the transfer-matrix method. Recently, the statistical distribution of the reflection coefficient has been studied in 1D by using a stochastic approach. ${ }^{8}$

\section{THE MODEL AND TRANSFER-MATRIX METHOD}

In a layer medium with normal incidence, the timeindependent Maxwell equations give the following coupled equations for the electric and magnetic fields, 


$$
\begin{aligned}
& \frac{\partial E(z)}{\partial z}=\frac{i \omega \mu(z)}{c} H(z), \\
& \frac{\partial H(z)}{\partial z}=\frac{i \omega \varepsilon(z)}{c} E(z),
\end{aligned}
$$

where $H$ denotes the $y$ component of the magnetic field, $E$ the $x$ component of the electric field, $\omega$ is the frequency of the wave, $c$ is the effective medium speed of the light, $\varepsilon(z)$ is the dielectric constant, and $\mu(z)$ is the magnetic permeability. Combining the above equations and setting $\mu(z)=1$ for a nonmagnetic medium, we have the following equation for the electric field,

$$
\frac{\partial^{2} E(z)}{\partial z^{2}}+\frac{\omega^{2}}{c^{2}} \varepsilon(z) E(z)=0 \text {. }
$$

Consider a randomly layered sample of $L$ layers with random dielectric constant and layer thickness. The dielectric constant $\varepsilon(z)$ contains both the real and imaginary parts, i.e., $\varepsilon(z)=\varepsilon^{\prime}(z)-i \varepsilon^{\prime \prime}(z)$. Here we assume that the real part $\varepsilon^{\prime}$ in the $n$th layer has the value $\varepsilon_{n}$ with $\varepsilon_{n}=1+\sigma \eta_{n}$, where $0<\sigma<1$ gives the amplitude of randomness, and $\eta_{n}$ is a random number between $[-1,1]$. The imaginary part is assumed to have a constant value $-\varepsilon^{\prime \prime}$ across the entire sample. Here a negative imaginary part denotes the coherent amplification of the field. The thickness $\Delta z$ of a given layer is assumed to follow an exponential distribution $a^{-1} \exp (-\Delta z / a), a$ being the mean layer thickness. The entire sample is embedded in a homogeneous medium with light speed $c$ and dielectric constant unity.

The solution of Eq. (3) in the $n$th layer has the form $E(z)=A_{n} \exp \left[i k_{n}\left(z-z_{n}\right)\right]+B_{n} \exp \left[-i k_{n}\left(z-z_{n}\right)\right]$, where $k_{n}$ is a complex wave vector and $z_{n}$ separates the $(n-1)$ th layer from the $n$ th. In the presence of gain, $\varepsilon^{\prime \prime}>0$, it is easy to show that the dispersion relation becomes

$$
k_{n}=\frac{\omega}{v_{n}}\left(1-i \frac{\beta_{n}}{\omega}\right),
$$

where

$$
\begin{aligned}
& \frac{\beta_{n}}{\omega}=\frac{\varepsilon_{n}}{\varepsilon^{\prime \prime}}\left\{\left[1+\left[\frac{\varepsilon^{\prime \prime}}{\varepsilon_{n}}\right]^{2}\right]^{1 / 2}-1\right\}, \\
& v_{n}^{2}=c^{2}\left[1-\left[\frac{\beta_{n}}{\omega}\right]^{2}\right] / \varepsilon_{n} .
\end{aligned}
$$

At the interface of two layers, both $E$ and $H$ fields are continuous. Since the medium is nonmagnetic $(\mu=1)$, the continuity of $H$ implies the continuity of the derivative of $E$ as can be seen from Eq. (2). ${ }^{9}$ Using these boundary conditions, the following transfer matrix is obtained:

$$
\left[\begin{array}{l}
A_{n+1} \\
B_{n+1}
\end{array}\right]=\tau^{(n)}\left[\begin{array}{l}
A_{n} \\
B_{n}
\end{array}\right] \equiv\left[\begin{array}{ll}
t_{11}^{(n)} & t_{12}^{(n)} \\
t_{21}^{(n)} & t_{22}^{(n)}
\end{array}\right]\left[\begin{array}{l}
A_{n} \\
B_{n}
\end{array}\right],
$$

with

$$
\begin{aligned}
& 2 t_{11}^{(n)}=\left(1+\frac{k_{n}}{k_{n+1}}\right) \exp \left(i k_{n} a_{n}\right), \\
& 2 t_{12}^{(n)}=\left(1-\frac{k_{n}}{k_{n+1}}\right) \exp \left(-i k_{n} a_{n}\right), \\
& 2 t_{21}^{(n)}=\left(1-\frac{k_{n}}{k_{n+1}}\right) \exp \left(i k_{n} a_{n}\right), \\
& 2 t_{22}^{(n)}=\left(1+\frac{k_{n}}{k_{n+1}}\right) \exp \left(-i k_{n} a_{n}\right),
\end{aligned}
$$

where $a_{n}$ is the thickness of the $n$th layer. From the products of these matrices, $\tau(L)=\prod_{n=0}^{L} \tau^{(n)}$, we obtain the transmission and reflection amplitudes of the sample as $t(L)=\left(\tau_{11} \tau_{22}-\tau_{12} \tau_{21}\right) / \tau_{22}$ and $r(L)=-\tau_{21} / \tau_{22}$, respectively.

Due to the amplification of the field in the sample, the flux is no longer a conserved variable. Using Eq. (3), it is easy to show that the sum of the reflection and transmission coefficients satisfies the following equality:

$$
\begin{aligned}
T(L)+R(L) & \equiv|t(L)|^{2}+|r(L)|^{2} \\
& =1+\frac{\varepsilon^{\prime \prime} \omega}{c} \int_{z_{0}}^{z_{L}}|E(z)|^{2} d z .
\end{aligned}
$$

Equation (7) recovers the unitary condition that $T+R=1$ when $\varepsilon^{\prime \prime}=0$. Knowing the function $T(L)$, the localization length $\xi$ can be obtained by taking the $L \rightarrow \infty$ limit of $\xi(L)$, where $1 / \xi(L)=-\ln [T(L)] /(2 L)$.

In the absence of gain, the wave localization behavior in a randomly layered medium has been studied previously. ${ }^{10}$ In the long-wavelength limit $(\lambda=2 \pi c / \omega \gg \rightarrow)$, the localization length $\xi_{0}$ is known to follow the $1 / \omega^{2}$ behavior. However, in the short-wavelength limit $(\lambda<a)$, $\xi_{0}$ crosses over to a constant minimum value. Although the localization length exhibits very different behaviors in two limits, nevertheless, the study of the power spectrum of the reflected noise has shown that the localization length $\xi_{0}$ is the only relevant length in such a system, independent of the wavelength or randomness. ${ }^{11}$ With the presence of the gain, the length that sets the amplification of the field also becomes relevant. This gain length $l_{g}$ is given by the inverse of the imaginary part of the wave vector in Eq. (4). In the small $\sigma$ and $\varepsilon^{\prime \prime}$ limit, we obtain from Eqs. (4) and (5) the expression for the gain length as $l_{g}=2 c / \varepsilon^{\prime \prime} \omega$. Thus, $\xi_{0}$ and $l_{g}$ are expected to be the only parameters in this 1D gain medium. It is also expected that the interaction between $\xi_{0}$ and $l_{g}$ will give rise to two new lengths. It is natural to guess that the localization length $\xi$ and the super-radiation threshold length $L_{\text {sr }}$ form the new length scales of the problem. In 1D, from the diffusion theory we have $L_{\mathrm{sr}} \cong 2.2\left(l_{g}\right)^{0.5}$. $^{4}$ However, it is also known that, in the long-wavelength limit, $\xi_{0} \cong(2-4) l .^{6,7}$ Thus, it is natural to replace the mean free path $l$ by $\xi_{0}$ in the expression of $L_{\mathrm{sr}}$, i.e., $L_{\mathrm{sr}} \cong\left(l_{g} \xi_{0}\right)^{0.5}$ and extend this length scale to the short-wavelength limit. It is shown in the following that the above assumptions of two-parameter scaling $\left(\xi_{0}\right.$ and $\left.l_{g}\right)$ and the existence of two new lengths $\left(L_{\mathrm{sr}}\right.$ and $\left.\xi\right)$ are indeed 
confirmed by our extensive numerical simulations. In what follows, we have set the values of light speed $c$ and averaged layer thickness $a$ to unity.

\section{RESULTS AND DISCUSSIONS}

For a given sample of $L$ layers, the probability density of the transmission and reflection coefficients are calculated at various wavelengths $\lambda$, degrees of randomness $\sigma$, and gain lengths $l_{g}$. In order to cover both the long- and short-wavelength limits, we have chosen $\lambda=1 / 2,1,10$, 25 , and $50 .{ }^{10}$ The values of randomness chosen are $\sigma=0.3,0.5$, and 0.9 . For each set of parameters, 10000 configurations are performed. In the absence of gain, the values of $\xi_{0}$ for the case of $\sigma=0.3$ are found to be $\xi_{0}=515,516,861,2630$, and 8900 for the corresponding $\lambda$ 's chosen above. In the long-wavelength limit, the analytic result $\xi_{0} \approx 12 /(\sigma \omega)^{2}$ gives $\xi_{0}=8438$ for $\lambda=50$. $^{10}$ Since this value is only $5 \%$ below the actual number, this is still in the regime of long wavelength. However, for $\lambda=25,10$, and 1 , the analytic formula gives $\xi_{0}=2109$, 338 , and 3.4 , respectively. This shows clearly the breakdown of the analytic formula as $\lambda$ becomes smaller. The crossover between two limits is apparent. The smallest value of $\xi_{0}$ in all the parameters considered here is $\xi_{0}=34$ for the case of $\lambda=\frac{1}{2}$ (or 1) and $\sigma=0.9$.

In the presence of gain, we introduce a dimensionless parameter $q^{-1}$ as the ratio of the gain length to the localization length to describe the amplification, i.e., $q=\xi_{0} / l_{g}{ }^{8}$ For a given value of $q$ and a fixed set of parameters $\lambda$ and $\sigma$, we have calculated the probability density for the reflection and transmission coefficients at various values of renormalized sample length $\Lambda=L / \xi_{0}$. The results for the case of $q=0.5$ are shown in Figs. 1 and 2 . In Fig. 1, we plot the probability density $P_{\Lambda}$ for the reflection coefficient as a function of a scaled variable $(R-1) / 2 q$ for three different values of $\Lambda$. At $\Lambda=0.3$,

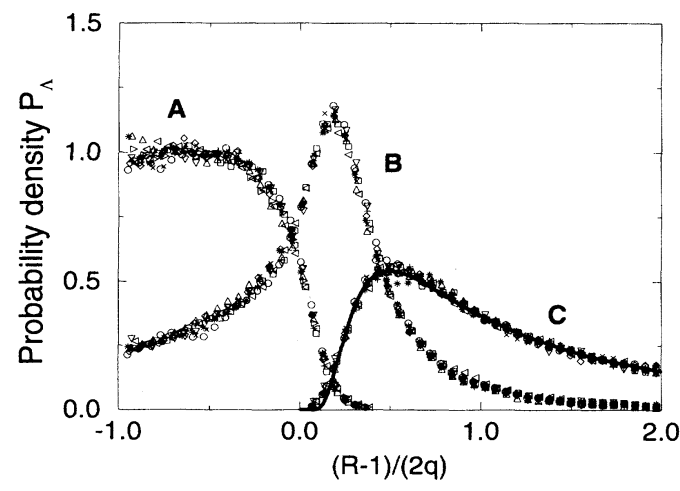

FIG. 1. For $q=0.5$, the probability density $P_{\Lambda}$ for the reflection coefficient $R$ is plotted as a function of the renormalized variable $(R-1) / 2 q$ at three renormalized lengths: $\Lambda=0.3$ (curve $A$ ), 0.6 (curve $B$ ), and 1.8 (curve $C$ ). Different symbols represent curves obtained from different sets of parameters $\lambda$ and $\sigma$ described in the text. The heavy line in curve $C$ is the steady-state distribution of Eq. (6).

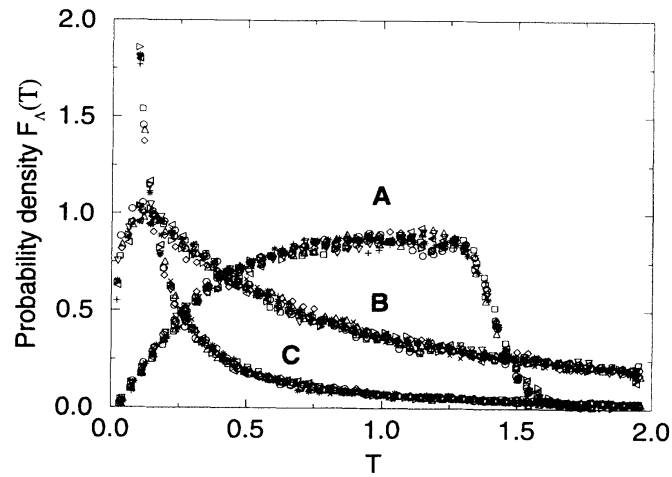

FIG. 2. For $q=0.5$, the probability density $F_{\Lambda}$ for the transmission coefficient $T$ is plotted at different renormalized lengths: $\Lambda=0.3$ (curve $A$ ), 0.6 (curve $B$ ), and 1.8 (curve $C$ ). Different symbols represent curves obtained from different sets of parameters $\lambda$ and $\sigma$ described in the text.

the data of $P_{0.3}$ calculated for all sets of parameters $\lambda$ and $\sigma$, except the case of $\xi_{0}=34$, fall into a single curve (curve $A$ ). Similarly, curves $B$ and $C$ give the distribution functions for $\Lambda=0.6$ and 1.8 , respectively. For the case of $\xi_{0}=34$, some deviations from curves $A, B$, and $C$ are observed. These deviations are due to the relative smallness of $\xi_{0}$. As the value of $\xi_{0}$ becomes comparable to the averaged layer thickness $a=1$, the scaling breaks down completely. In curve $C$, we also plot the analytic result $P_{\infty}$ obtained from the stochastic Riccati equation in the white-noise model for an infinite sample. ${ }^{8}$ This result,

$$
P_{\infty}\left(\frac{R-1}{2 q}\right)=\left(\frac{2 q}{R-1}\right)^{2} \exp \left(\frac{-2 q}{R-1}\right),
$$

is shown by a heavy solid line in curve $C$. The excellent agreement between the numerical data of $P_{1.8}$ and the analytic $P_{\infty}$ indicates that a steady-state distribution is reached when $\Lambda$ has the value 1.8. Indeed, with the further increment of $\Lambda$, we do not find any significant change of the distribution. Besides the case of $q=0.5$, we have also studied the cases of $q=0.1,0.2,1,2.7,5$, and 10. The scaling of $\xi_{0}$ discussed above holds for all values of $q$ considered. Although the distribution function $P_{\Lambda}$ has different forms as $q$ varies at small $\Lambda$, they all converge to $P_{\infty}$ of Eq. (8) after reaching some saturation length $\Lambda_{s}(q)$. The corresponding results of the transmission coefficient are shown in Fig. 2. At $q=0.5$, the probability density $F_{\Lambda}$ is plotted as a function of $T$ for $\Lambda=0.3$ (curve $A$ ), 0.6 (curve $B$ ), and 1.8 (curve $C$ ). Except for the case of $\xi_{0}=34$, the scaling of $\xi_{0}$ holds in the transmission case too. Since the transmission disappears in an infinite sample, the trivial saturation distribution of $F_{\infty}$ is a delta function at $T=0$. However, what is more interesting is that, as a function of $\Lambda$, the averaged transmission coefficient $\langle T(\Lambda)\rangle$ is an increasing function of $\Lambda$ at small $\Lambda$ due to the amplification effect. It reaches a maximum at some $\Lambda_{T}$ and then dies off as $\Lambda \rightarrow \infty$ because of the localization effect. Thus, the existence of $\Lambda_{T}$ is a result of 
competition between amplification and localization effects.

To determine the saturation length $\Lambda_{s}$, we use two different criteria. First we compute the standard deviation, $\Delta_{\Lambda}$, between $P_{\Lambda}(x)$ and $P_{\infty}(x)$ of Eq. (8) in the interval $x \equiv(R-1) / 2 q=[0,10]$ as a function of $\Lambda$. We define the saturation length $\Lambda_{s}$ as the value of $\Lambda$ where $\Delta_{\Lambda}$ is within $15 \%$ of its saturation value. As a complementary test, we also estimate the saturation in the large $R$ limit by considering the distribution in the reciprocal variable $y \equiv x^{-1}=2 q /(R-1)$. Both criteria give consistent results.

In Fig. 3, we plot the saturation length $\Lambda_{s}$ obtained from the reflection coefficient (solid circles) and the maximum transmission length $\Lambda_{T}$ (solid triangles) from $q=0.1$ to 10 in logarithmic scales. The data of the solid triangles have similar error bars as those of the solid circles. Both sets of data agree well with the function $q^{-0.5}$, which is shown by a dashed line. From $q=\xi_{0} / l_{g}$ and $\Lambda=L / \xi_{0}$, both $L_{S}$ and $L_{T}$ are indeed the manifestation of the super-radiation threshold length $L_{\mathrm{sr}} \cong\left(l_{g} \xi_{0}\right)^{0.5}$. These results also demonstrate that the replacement of $l$ by $\xi_{0}$ in fact extends the manifestation of $L_{\mathrm{sr}}$ from the long-wavelength limit to the short-wavelength limit.

The amplification of the coherent backscattering effect may also reduce the localization length in a gain medium. A simple expression for the localization length can be derived from the flux production relation of Eq. (7) and probability density $P_{\infty}$ of Eq. (8). We assume that both the right-going $A(z)$ and left-going $B(z)$ of the field $E(z)$ decay exponentially in magnitude, i.e., $|A(z)|^{2}$ $=\exp \left[2\left(z_{0}-z\right) / \xi_{i}\right]$ and $|B(z)|^{2}=R_{i} \exp \left[2\left(z_{0}-z\right) / \xi_{i}\right]$. Here the subscript $i$ denotes a particular configuration. Substituting these expressions for $|A(z)|^{2}$ and $|B(z)|^{2}$ into Eq. (7) and integrating from $z_{0}=0$ to $z_{L}=\infty$, we find

$$
R_{i} \approx 1+\frac{\varepsilon^{\prime \prime} \omega}{c}\left(R_{i}+1\right) \int_{0}^{\infty} \exp \left(-2 z / \xi_{i}\right) d z
$$

Here, we have ignored the contributions from the cross

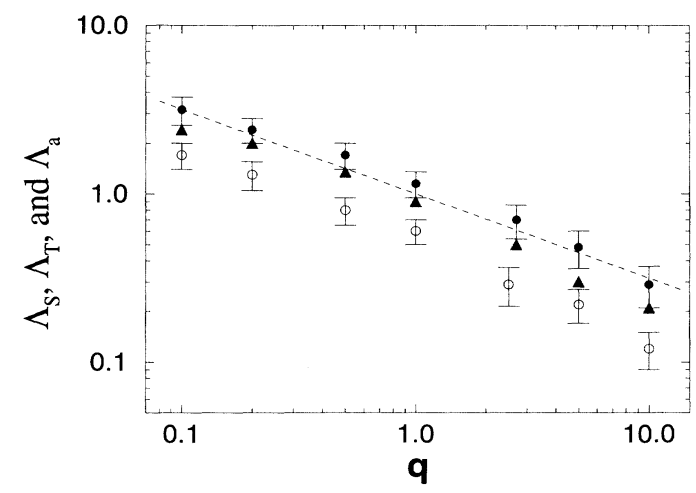

FIG. 3. The saturation length $\Lambda_{s}$ (or $\Lambda_{a}$ ) for the reflection coefficient in a gain (or dissipative) medium is plotted as a function of $q$ as solid circles (or open circles). The solid triangles represent the maximum transmission length $\Lambda_{T}$ in a gain medium. The dashed line shows the function $q^{-1 / 2}$. terms $\left[A(z)^{*} B(z)+A(z) B(z)^{*}\right]$ in the integral of Eq. (7). Since the phases of $A(z)$ and $B(z)$ vary randomly from layer to layer, it is plausible to assume that their contribution becomes negligible after integration. After performing the integration, Eq. (9) can be rearranged in the form

$$
\frac{1}{\xi_{i}}=\frac{\varepsilon^{\prime \prime} \omega}{2 c}\left[1+\frac{2}{R_{i}-1}\right]
$$

By taking the configurational average on both sides of Eq. (10) and using the relation that $\left\langle 1 /\left(R_{i}-1\right)\right\rangle$ $=1 / 2 q$ obtained from Eq. (8), we obtain $\xi \equiv\left\langle 1 / \xi_{i}\right\rangle^{-1}=\xi_{0} /(1+q)$, or $1 / \xi=1 / \xi_{0}+1 / l_{g}$. Numerically, the localization length is obtained by taking the configurational average of $1 / \xi_{i}(L)=-\ln \left[T_{i}(L)\right] / 2 L$. In Fig. 4 we plot the renormalized localization length $\xi_{\Gamma}(1+q) / \xi_{0}$ as a function of renormalized length $\Gamma=L /\left(l_{g} \xi_{0}\right)^{0.5} \equiv \Lambda q^{0.5}$, where $\xi_{\Gamma} \equiv\left\langle 1 / \xi_{i}(\Gamma)\right\rangle^{-1}$. We have renormalized the length $L$ by the saturation length so that the localization length $\xi_{\Gamma}$ will converge in a similar rate to its saturation value as $\Gamma \rightarrow \infty$ for different $q$ 's. The results of $q=0.5,1$, and 5 are plotted in curves $A, B$, and $C$, respectively. Different symbols in a curve represent different sets of parameters of $\lambda$ and $\sigma$. These curves show again the scaling of $\xi_{0}$ for a given $q$. In the large $\Lambda$ limit, all three curves approach unity. This confirms our prediction of $1 / \xi=1 / \xi_{0}+1 / l_{g}$. The reduction of localization length shown in this simple relation clearly demonstrates the amplified effect of the coherent backscattering in a gain medium that enhances the wave localization. For $q=1$ and $5, \xi_{\Gamma}$ becomes negative at small $\Gamma$ because of $\left\langle\ln \left[T_{i}(\Gamma)\right]\right\rangle>0$. Although curves $A$, $B$, and $C$ behave differently at small $\Gamma$, they all show a maximum change around $\Gamma \cong 1$.

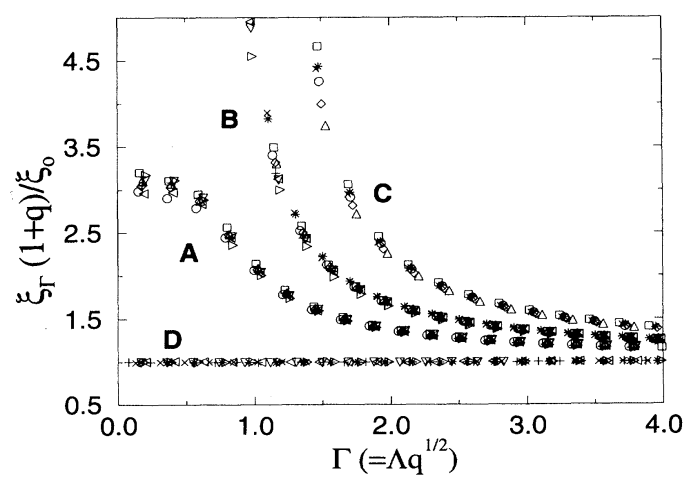

FIG. 4. The renormalized localization length $\xi_{\Gamma}(1+q) / \xi_{0}$ is plotted as a function of renormalized length $\Gamma=\Lambda q^{0.5}$. Curves $A, B$, and $C$ are for $q=0.5,1$, and 5, respectively. Different symbols represent curves obtained from different sets of parameters $\lambda$ and $\sigma$ described in the text. Curve $D$ shows the data obtained from a dissipative medium. 


\section{THE CASE OF DISSIPATIVE MEDIA}

Finally, we have also carried out similar calculations for dissipative media. In this case the imaginary part of the dielectric constant becomes positive with $\varepsilon^{\prime \prime}<0$. An attenuation length can be defined as $l_{a}=-2 c / \varepsilon^{\prime \prime} \omega$. For any given $q=\xi_{0} / l_{a}$, the scaling of $\xi_{0}$ is again observed in the probability density for both the transmission and reflection coefficients. In the large $\Lambda$ limit, the probability density for the reflection coefficient approaches a steady-state distribution ${ }^{8}$

$P_{\infty}(R)=2 q \exp (2 q) \exp [-2 q /(1-R)] /(1-R)^{2}$

The saturation length $\Lambda_{a}$ for different values of $q$ is plotted with open circles in Fig. 3. Again, a $\left(l_{a} \xi_{0}\right)^{0.5}$ behavior is seen. However, the averaged transmission coefficient is a monotonic decaying function of $\Lambda$. The localization length can be obtained in a similar way as described above for the case of amplification. Equations (9) and (10) are still valid except that $\varepsilon^{\prime \prime}$ has a negative value now. The average value of $1 /\left(1-R_{i}\right)$ can be obtained from the distribution function Eq. (11) and has the expression $\left\langle 1 /\left(1-R_{i}\right)\right\rangle=1+1 / 2 q$. Substituting this relation into Eq. (10), we find again $\xi \equiv\left\langle 1 / \xi_{i}\right\rangle^{-1}=\xi_{0} /(1+q)$, or $1 / \xi=1 / \xi_{0}+1 / l_{a}$. In fact, such a relation for the dissipative medium has been suggested previously without any derivation. ${ }^{12}$ Numerically, we have randomly picked some sets of data and plotted the localization length, renormalized by $\xi_{0} /(1+q)$, as a function of $\Lambda q^{0.5}$ in curve $D$ of Fig. 4. This is apparent that our derived simple relation for $\xi$ is indeed valid.

\section{CONCLUSIONS}

In conclusion, we have studied the statistics of reflection and transmission coefficients and the localization behavior in randomly layered media with gain or dissipation. In both cases it is found that only two parameters are relevant, $\xi_{0}$ and $l_{g}$ (or $l_{a}$ ), independent of the randomness or the wavelength. This is consistent with the previous study of the reflected noise power spectrum, where the localization length $\xi_{0}$ is the only relevant parameter in describing the wave transport property in one dimension, not the wavelength or the randomness independently. The interplay of these two parameters gives rise to two new lengths. In the case of gain, both the saturation length of the reflection coefficient and the length for the maximum transmission behave like $\left(l_{g} \xi_{0}\right)^{0.5}$. This is the manifestation of the super radiation, which is largely suppressed as a result of localization. The localization length is reduced in the presence of gain due to the enhancement of the coherent backscattering effect. The localization length is found to follow a simple relation $1 / \xi=1 / \xi_{0}+1 / l_{g}$. In the case of dissipation, similar behaviors are found for the saturation length of the reflection coefficient and the localization length if we replace the gain length $l_{g}$ by the attenuation length $l_{a}$. Despite all these similarities, there are some major differences. Unlike the case of gain, the averaged transmission coefficient is a monotonic decreasing function of the sample size and does not have a peak. The sum of the transmission and reflection coefficients is smaller than 1 in a dissipative medium while larger than 1 and unbounded in a gain medium.

The author thanks P. Sheng, S. Feng, N. Kumar, and, particularly, K. M. Yoo for fruitful discussions.
${ }^{*}$ Also at the Institute of Physics, Academia Sinica, Beijing, China.

${ }^{1}$ For example, see Scattering and Localization of Classical Wave in Random Media, edited by P. Sheng (World Scientific, Singapore, 1990).

${ }^{2}$ N. M. Lawandy, R. M. Balachandran, A. S. L. Gomes, and E. Sauvain, Nature 368, 436 (1994).

${ }^{3}$ A. Z. Genack and J. M. Drake, Nature 368, 400 (1994).

${ }^{4}$ V. S. Letokhov, Zh. Eksp. Teor. Fiz. 53, 1442 (1967) [Sov. Phys. JETP 26, 835 (1968)].

${ }^{5}$ A. Yu. Zyuzin, Europhys. Lett. 26, 517 (1994); also S. C. Feng and Z. Q. Zhang (unpublished).

${ }^{6}$ Q. J. Chu and Z. Q. Zhang, Phys. Rev. B 39, 7120 (1989).

${ }^{7}$ E. N. Economou, C. M. Soukoulis, and A. D. Zdetsis, Phys.
Rev. B 30, 1686 (1984).

${ }^{8}$ P. Pradhan and N. Kumar, Phys. Rev. B 50, 9644 (1994).

${ }^{9}$ Notice that Eq. (2) is derived only for the case of normal incidence in a layered medium. The derivative of the tangential component of the electric field is not continuous across the boundary in general, like the case of an oblique incidence or arbitrary interface. [See, for example, A. Moroz, J. Phys. Condens. Matter 6, 171 (1994).]

${ }^{10} \mathrm{P}$. Sheng, B. White, Z. Q. Zhang, and G. Papanicolaou, Phys. Rev. B 34, 4757 (1986).

${ }^{11}$ P. Sheng, Z. Q. Zhang, B. White, and G. Papanicolaou, Phys. Rev. Lett. 57, 1000 (1986); 59, 1918 (1987).

${ }^{12}$ A. R. McGurn, K. T. Christensen, and F. M. Mueller, Phys. Rev. B 47, 13120 (1993). 